PACS 72.20.Lw, 72.20.Pa

\title{
Size effects in p-PbTe nanostructures on polyamide
}

\author{
D.M. Freik, I.K. Yurchyshyn, V.Yu. Potyak, Yu.V. Lysiuk \\ Physical-chemical Institute at the Vasyl Stefanyk PreCarpathian National University \\ 57, Shevchenko str., 76025 Ivano-Frankivsk, Ukraine \\ E-mail: freik@pu.if.ua, igoor2010@gmail.com,gucul19@gmail.com
}

\begin{abstract}
The thickness dependences of the thermoelectric parameters were observed at room temperature for nanostructures $p$ - $\mathrm{PbTe}$, grown from the vapor phase on polyamide film substrates. An attempt to explain detected dependences in the range of quantum theory was done.
\end{abstract}

Keywords: electrical properties, thermoelectricity, nanostructures.

Manuscript received 22.02.11; accepted for publication 14.09.11; published online 21.09.11.

\section{Introduction}

At the present stage of developing the materials for thermoelectric applications, low-dimensional systems are the main object of scientific community researches. We know that Wiedemann-Franz law limits variation of Seebeck coefficient $(S)$, electro- $(\sigma)$ and thermal conductivity $(\chi)$ to increase value of the material thermoelectric figure of merit $Z=S^{2} \sigma / \chi$. When dimensionality of a system decreases from 3dimensional crystalline solids to 2-dimensional (quantum wells), 1-D (quantum wires) and finally to 0-D (quantum dots), there arise new physical phenomena that can create additional opportunities for independent variation $S, \sigma$, and $\chi[1]$.

For accurate interpretation and prediction of kinetic parameters behavior in the periodic semiconducting nanostructure, it is necessary to know the energy spectrum and properties of its separate structural unit. In quantum wells $(\mathrm{QWs})$, movement of charge carriers is confined in one direction. This leads to quantization of the quasi-momentum and carrier energy spectrum in this direction, which determines quantum-mechanical scenario for various physical phenomena.

Semiconductor compounds IV-VI are well known as materials for optoelectronic, thermoelectric and other applications. Recently, interest in them has increased due to theoretical predictions and further observation of substantial growth of thermoelectric figure of merit in superlattices, based on compounds IV-VI [2,3]. It should be borne in mind that research on thermoelectric properties of these compounds inevitably leads to difficulties associated with oxidation processes that occur in them in the air. It was found in $[4,5]$ that, in general, diffusion processes in $\mathrm{PbTe}$ nanostructures lead to an increase of $p$-type carrier concentration and reduction of $n$-type one. This causes the main type sign inversion from $n$ to $p$ in the case of nanostructures being initially of the $n$-type. If the investigated object is the $n$ type condensate, this problem can be solved by film coating protective layer EuS. The authors of [6] have shown that EuS thickness $\sim 30 \mathrm{~nm}$ is quite enough to completely avoid the oxygen action. Thus, this protective layer is regarded as one of barriers that creates a quantum well for carriers in $\mathrm{PbTe}$ structure. Assuming that oxidative processes lead to the formation of a dielectric layer on the condensate surface, this layer can be considered as an external barrier in the quantum well structure. Researching $\mathrm{PbTe}$ nanostructures of $p$-type and considering the acceptor effect of oxygen, it is possible to study samples without any protective layer, and this simplifies the process of their creation.

Due to high technological importance of lowdimensional structures, their properties are the subject of many research groups. For example, in the study of thickness $(d)$ dependences of thermoelectric parameters for $\mathrm{PbSe}$ in quantum well structure $\mathrm{KCl} / \mathrm{PbSe} / \mathrm{EuS}$ [7] experimental data show clearly non-monotonous, oscillation character curves, what indicates the presence of size quantization. The authors [8] examined samples of $n$-type $\mathrm{KCl} / \mathrm{PbS} / \mathrm{EuS}$. The complex nature of $d$ dependences for thermoelectric parameters has been explained by competition between size quantization and percolation phenomenon. This shows that selforganization processes strongly influence the thickness dependence of kinetic parameters in nanostructures. In 
[9] it was found that for heterostructure $n-\mathrm{PbTe} / p$ $\mathrm{SnTe} / n$-PbTe oscillatory $d$-dependences of thermoelectric parameters are associated with size quantization of light hole gas in SnTe quantum well with walls of a limited height.

A qualitatively similar result was obtained by authors [10] for samples $\mathrm{KCl} / \mathrm{PbTe} / \mathrm{EuS}$-type of two series: stoichiometric $\mathrm{PbTe}$ and that containing 2 at.\% $\mathrm{Pb}$. Changing the lead content in samples, they varied the $n$ type carrier concentration level. This allowed fixing the extremum shift in thickness dependences of thermoelectric parameters for samples with different carrier concentrations. Such approach suggests that in the case of $p$-type material, to get different main carrier concentrations, it is possible to consider the same samples only with different time exposure to air.

The aim of this work is to study some features in thickness dependences behavior of thermoelectric parameters for $p$-type lead telluride nanostructures with different carrier concentrations, deposited on substrate with polyamide tape.

\section{Experimental}

Samples for investigation were grown from the vapor phase by open evaporation in vacuum on substrates with polyamide tape of $\mathrm{PM}-1$ type. The evaporator temperature during deposition was $T_{e}=700{ }^{\circ} \mathrm{C}$ and substrate temperature $T_{s}=150{ }^{\circ} \mathrm{C}$.
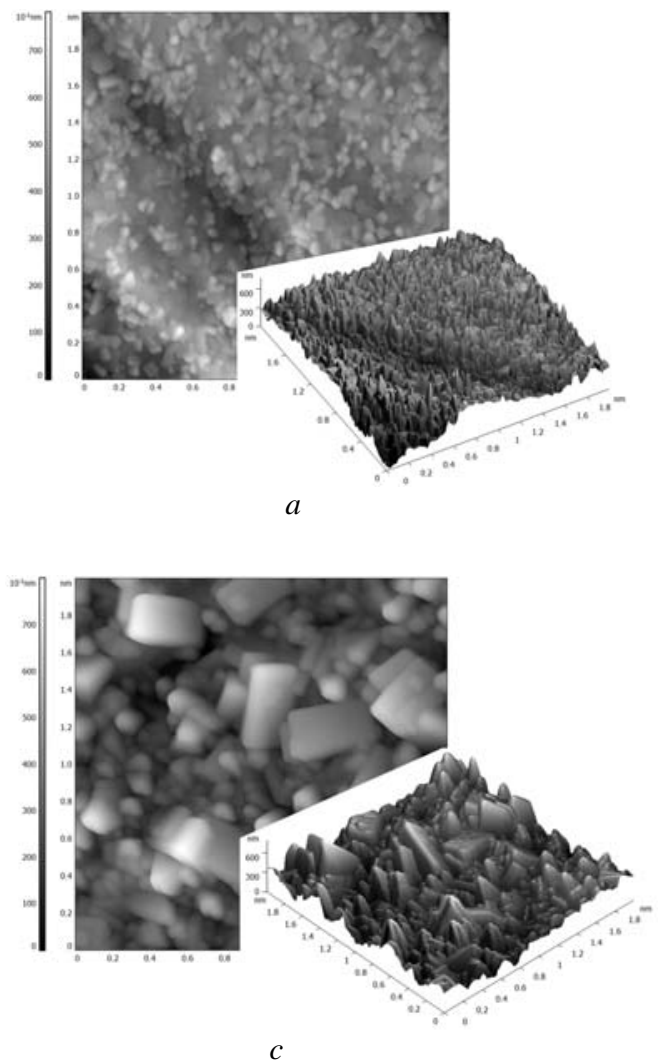

The obtained nanostructures were investigated using the methods of atomic-force microscopy (AFM) with Nanoscope 3a Dimension 3000 (Digital Instruments, USA) in the periodic contact mode. Measurements were made in the central part of samples using serial silicon probes NSG-11 with the nominal edge rounding radius to $10 \mathrm{~nm}$ (NT0MDT, Russia).

Measurements of electrical parameters were carried out at room temperature in air and under constant magnetic field. The measured sample had four Hall and two current contacts. As ohmic contacts, silver films were used. The current through the sample was $\sim 100 \mu \mathrm{A}$. The induction of magnetic field normal to the condensate surface was $2 \mathrm{~T}$.

We investigated two series of samples: as-grown and those aged in air for 50 hours.

The Hall mobility was calculated as $\mu=R_{\mathrm{H}} \cdot \sigma$. The $p$-type carrier concentration was determined using the ratio $p=A /\left(R_{\mathrm{H}} \cdot e\right)$, considering it is appropriate to real concentrations by far predominant one type of carriers and taking the Hall factor $A=1$. All the samples demonstrated $p$-type conductivity.

\section{Results}

Results of atomic force microscopy show that $\mathrm{PbTe}$ on polyamide has a clear island-like structure (Fig. 1). Thus, with increasing the deposition time (thickness of the condensate) the size of nanostructures in lateral and
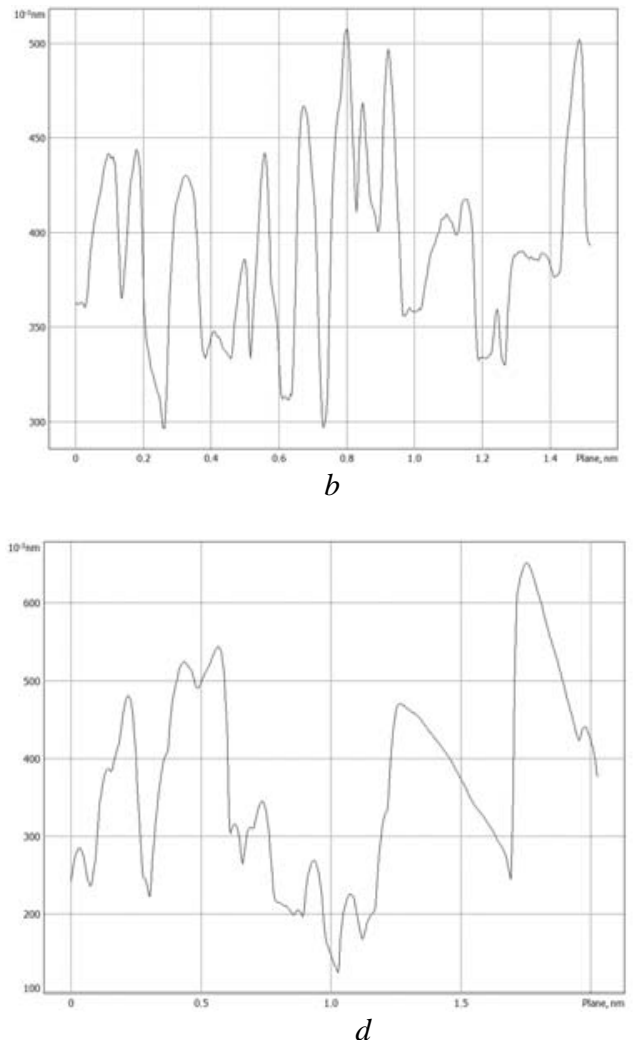

Fig. 1. ACM images $(a, c)$ and profilograms $(b, d)$ for nanostructures $\mathrm{PM}-1 / p-\mathrm{PbTe}$ obtained at various deposition times $t$, min: $5(\mathrm{a}, \mathrm{b}), 30(\mathrm{c}, \mathrm{d})$.

\section{(C) 2011, V. Lashkaryov Institute of Semiconductor Physics, National Academy of Sciences of Ukraine}


vertical directions increases. In previous works [11, 12], it was found that lead chalcogenides grow in accord with the Volmer-Weber mechanism on dielectric substrates such as $\mathrm{KCl}, \mathrm{NaCl}$ and others. Obviously, in this case the polyamide is not an exception. However, the properties of nanostructures are highly dependent on technological parameters of growth (substrate temperature, condensation rate, etc.).

Fig. 2 shows the dependence of conductivity on $\mathrm{PbTe}$ layer thickness in $\mathrm{PM}-1 / \mathrm{PbTe}$ nanostructure with different time exposures to air. At the thickness of $\approx 370 \mathrm{~nm}$, we observed a clear maximum for all the series. Also, we have a sharp increase of conductivity, ranging from $150 \mathrm{~nm}$ to less thicknesses (Fig. 2a).

For the Hall coefficient in thickness dependences (Fig. 1b), it is observed two distinct maxima at $\sim 160$ and $540 \mathrm{~nm}$. Thus, for two variables $\sigma$ and $R_{\mathrm{H}}$ in their $d$ dependences peak shifts occur to the left with growth of the $p$-type carrier concentration (Fig. $2 \mathrm{a}$ and $2 \mathrm{~b}$ ).

The thickness dependences for carrier mobility show a clear minimum (Fig. 2c). For as-grown structures $x_{\min }=200 \mathrm{~nm}, x_{\max }=640 \mathrm{~nm} \quad$ (Fig. 2c, curve 1). For other two - these values are approximately equal to: $x_{\min }=170 \mathrm{~nm}, x_{\max }=450 \mathrm{~nm} \quad$ (Fig. 2c, curves 2,3), respectively.

For concentrations, we revealed two minima and one maximum: for as-grown structures $x_{\min 1}=160 \mathrm{~nm}$, $x_{\min 2}=570 \mathrm{~nm}, x_{\max }=270 \mathrm{~nm}$ (Fig. 2d, curve 1) for other two $-x_{\min 1}=170 \mathrm{~nm}, \quad x_{\min 2}=540 \mathrm{~nm}, \quad x_{\max }=280 \mathrm{~nm}$ (Fig. 2d, curves 2 and 3).

Since the sudden change in the main type carrier concentration has place at the initial stages of exposure
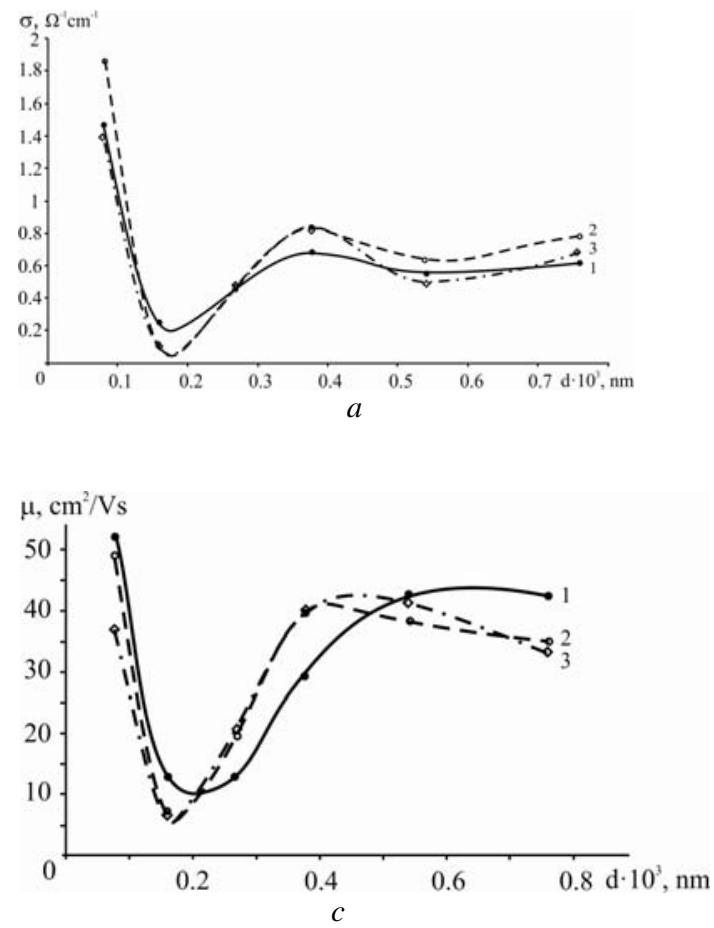

Fig. 2. Dependence of conductivity $\sigma(\mathrm{a})$, Hall coefficient $R_{\mathrm{H}}(\mathrm{b})$, mobility $\mu$ (c) and main type carrier concentration $n(d)$ on the thickness of PbTe nanostructures on polyamide at $T=300 \mathrm{~K}$ for various times of exposure to air $t: 1-$ as-grown, $2-24 \mathrm{~h}$, $3-50 \mathrm{~h}$.

(C) 2011, V. Lashkaryov Institute of Semiconductor Physics, National Academy of Sciences of Ukraine to air [4], then at prolonged exposure we do not fix the apparent relative extrema shift of thickness dependence for $\mathrm{PbTe}$ nanostructures on polyamide (Fig. 2).

Seebeck coefficient (Fig. 3a) displays two distinct maxima $x_{\max 1} \approx 120 \mathrm{~nm}, \quad x_{\max 2} \approx 380 \mathrm{~nm}$ and one minimum $x_{\min } \approx 270 \mathrm{~nm}$. Here we also find the maxima shift to the left with increasing the $p$-type carrier concentration.

Based on the values of $S(d)$ and $\sigma(d)$ (Figs $2 \mathrm{a}$ and $3 \mathrm{a})$, the dependence of power factor $P=S^{2} \sigma$ on the thickness of PbTe with different carrier concentrations is determined for structures $\mathrm{PM}-1 / \mathrm{PbTe}$ (Fig. $3 \mathrm{~b}$ ). There is a sharp thermoelectric power factor increase with reducing the thickness of structures, starting with $\sim 200 \mathrm{~nm}$.

\section{Discussion}

According to experimental findings (Figs 2 and 3), thickness dependences of thermoelectric parameters for $p$-PbTe structures on polyamide have the explicit oscillatory character. Naturally, we assumed that this behavior is due to size effects in quantum well formed by the potential barrier on the edge of polyamide substrate and oxidative layer on the condensate surface (similar to the results of [7-10]).

For the case of infinitely high barriers, electronic confinement leads to quantization of the quasimomentum transverse component and to formation of energy subbands in lateral direction. For infinitly height barriers, using effective mass approximation, energy levels are given [10]:
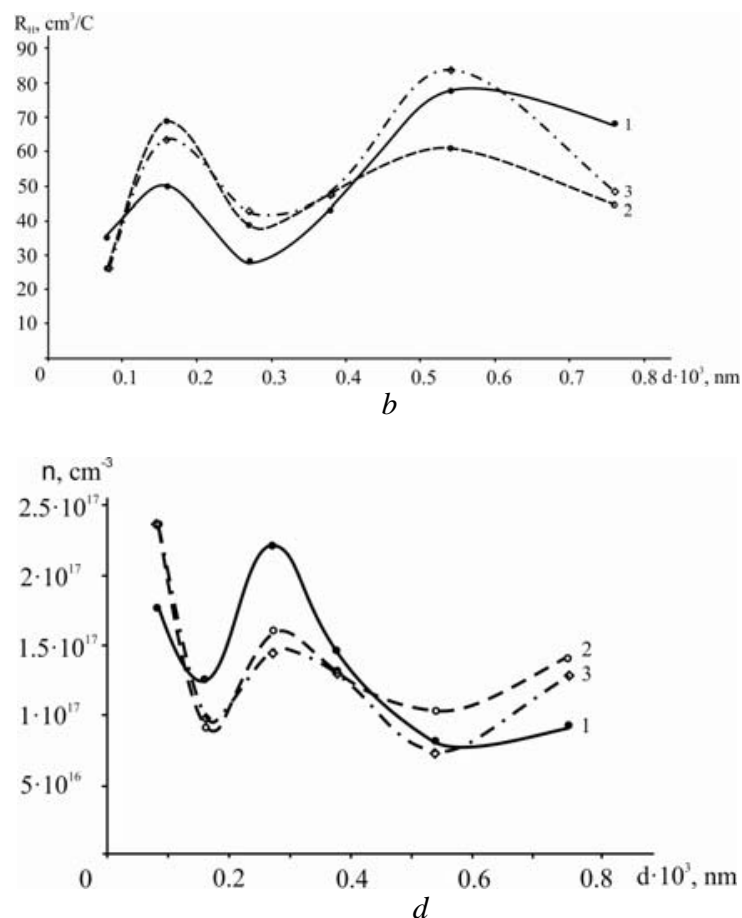
Table. Values of the oscillation period $\left(\Delta d_{\text {exp }}\right)$, effective mass $\left(m_{z}^{*}\right)$ and Fermi energy $\left(\varepsilon_{F}\right)$ calculated on their basis for nanostructures based on IV - VI compounds.

\begin{tabular}{|l|c|c|c|c|}
\hline \multicolumn{1}{|c|}{ Structures } & $\Delta d_{\text {exp }}, \mathrm{nm}$ & $m_{z}{ }^{*}$ & Fermi energy $\varepsilon_{\mathrm{F}}, \mathrm{eV}$ & Literature \\
\hline $\mathrm{KCl}(001) / n-\mathrm{PbS} / \mathrm{EuS}$ & 55.6 & $0.08 m_{e}$ & $1.5 \cdot 10^{-3}$ & {$[8]$} \\
\hline $\mathrm{KCl}(001) / n-\mathrm{PbSe} / \mathrm{EuS}$ & 59 & $0.04 m_{e}$ & $2.7 \cdot 10^{-3}$ & {$[7]$} \\
\hline $\mathrm{KCl}(001) / n-\mathrm{PbTe} / \mathrm{EuS}$ & 100 & $0.024 m_{e}$ & $1.56 \cdot 10^{-3}$ & {$[10]$} \\
\hline $\begin{array}{l}\mathrm{KCl}(001) / n-\mathrm{PbTe} \\
\text { with } 2 \text { at. } \% \mathrm{~Pb} / \mathrm{EuS}\end{array}$ & 132 & $0.024 m_{e}$ & $8.98 \cdot 10^{-4}$ & $10]$ \\
\hline polyamide $/ p-\mathrm{PbTe}$ & 350 & $0.022 m_{e}$ & $1.4 \cdot 10^{-4}$ & our data \\
\hline mica $/ p-\mathrm{SnTe}$ & 400 & $0.12 m_{e}$ & $1.9 \cdot 10^{-5}$ & our data \\
\hline$n-\mathrm{PbTe} / p-\mathrm{SnTe} / n-\mathrm{PbTe}$ & 3 & $0.12 m_{e}$ & 0.5 & {$[9]$} \\
\hline
\end{tabular}

$E=\frac{\hbar^{2}}{2 m_{z}^{*}} \frac{\left.\right|_{1} ^{2}}{d_{Q W}^{2}} N^{2}+\frac{\hbar^{2} k_{x}^{2}}{2 m_{x}^{*}}+\frac{\hbar^{2} k_{y}^{2}}{2 m_{y}^{*}}$,

where $m_{z}^{*}$ is the effective mass for movement perpendicular to the QW; $k_{x}, k_{y}$ and $m_{x}^{*}, m_{y}^{*}-$ components of the wavevector and effective mass, respectively, for movement parallel to the QW. Formula (1) shows that with decreasing QW thickness $\left(d_{Q W}\right)$, ground state energy $(N=1)$ shifts to higher values.

Since $\mathrm{PbTe}$ is degenerate semiconductor, the quantization condition is expressed through the Fermi wave $\lambda_{\mathrm{F}}$ :

$\lambda_{\mathrm{F}}=\frac{h}{\sqrt{2 m_{\mathrm{Z} \mid \mathrm{F}}^{*} \mathrm{~F}}}$,

and the number of occupied subbands in a smooth QW with thickness $d_{Q W}$ is equal

$N=\frac{2 d_{Q W}}{\lambda_{\mathrm{F}}}=\frac{2 d_{Q W}}{h} \sqrt{2 m_{\mathrm{Z}}^{*} \mid \mathrm{E}_{\mathrm{F}}}$,

the period of oscillation is determined by the relation:

$\Delta d=\frac{\lambda_{\mathrm{F}}}{2}=\frac{h}{\sqrt{8 m^{*}{ }_{1} \mathrm{E}_{\mathrm{F}}}}$.

Here, $\varepsilon_{\mathrm{F}}$ is the Fermi energy.

Note that due to a relatively thick oxidative surface layer the QW thickness $\left(d_{Q W}\right)$ can be significantly less than the thickness of nanostructure (d). From (3), (4), it follows that at a fixed QW thickness the Fermi level $\left(\varepsilon_{\mathrm{F}}\right)$ increase leads to an increase in the number of populated subbands $(N)$ and to a decrease in the oscillation period $(\Delta d)$.

Oscillatory behavior in thickness dependences of thermoelectric parameters is confirmed by the theoretical work [13], what assumes parabolicity of energy bands and infinite depth of the well. But it should be noticed that experimental oscillation amplitudes in $d$ dependences of thermoelectric parameters are much more large than the theoretical one. We consider that this difference has place because of assumption of infinite barrier height, what is confirmed by the data in [14], where for $\mathrm{PbTe} / \mathrm{Pb}_{1-\mathrm{x}} \mathrm{Eu}_{\mathrm{x}} \mathrm{Te}$ quantum wells it was found that energy barrier height reduction leads to increase of the thermoelectric power factor $S^{2} \sigma$.

The authors $[7,8]$ offer to determine a theoretical oscillation period value $\left(\Delta d_{\text {theor }}\right)$ for nanostructures, based on IV-VI compounds, using a known Fermi energy $\varepsilon_{\mathrm{F}}$ of single crystals. In our opinion, for nanostructures Fermi level is largely dependent on their topology and size, which, in turn, are determined by technological factors of growth. Therefore, for barrier nanostructures it is more justifiably to evaluate their energy characteristics using the oscillation periods values $\left(\Delta d_{\text {exp }}\right)$ of thermoelectric parameters, determined in the experiment (Figs 2 and 3 ). Table shows Fermi energy values for the series of nanostructures that are investigated in [7-10] and our data, evaluated by
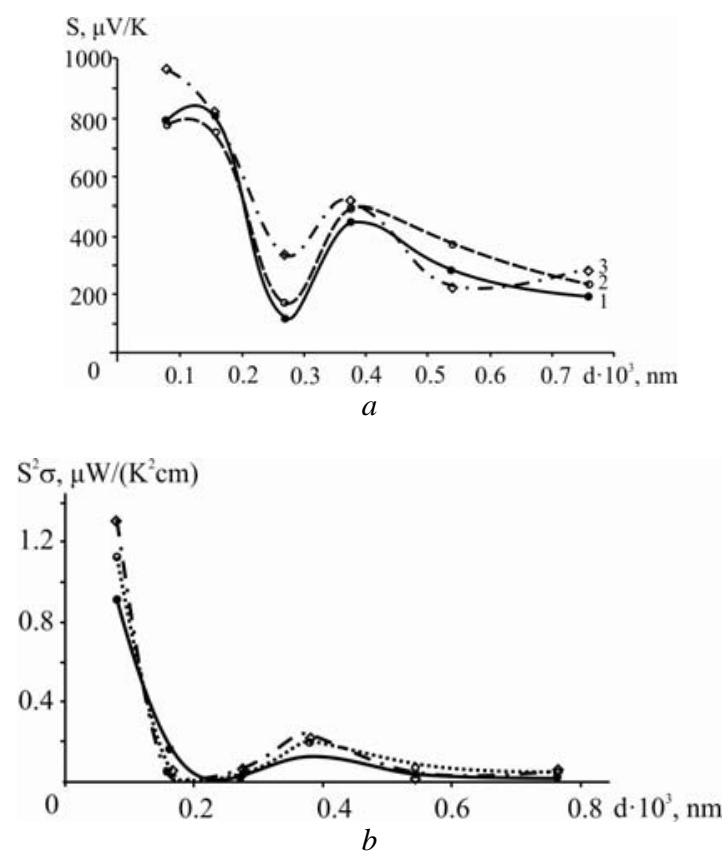

Fig. 3. Dependence of Seebeck coefficient $S($ a) and thermoelectric power $S^{2} \sigma(b)$ on the thickness of $\mathrm{PbTe}$ nanostructures on polyamide at $T=300 \mathrm{~K}$ for various times of exposure to air $t$ : 1 - as-grown, $2-24 \mathrm{~h}, 3-50 \mathrm{~h}$. 
substitution of experimental values $\left(\Delta d_{\exp }\right)$ for the oscillation period to Eq. (4). Note that the determined values $\varepsilon_{\mathrm{F}}$ are significantly different from Fermi energy values for massive single crystals [15]. This confirms the need to consider other reasons that affect on size effects in these structures. In our case, they are the size and topological differences of separate nanocreations, which depend on the thickness of $p$-PbTe nanostructure on polyamide (Fig. 1). Assuming that oxygen diffusion is accompanied by formation of thin adsorbed layer on the surface of each nanocrystal, the energy spectrum quantization of holes takes place within this crystallite.

More correct, from the perspective of the proposed modeling approach for analysis of barrier quantumdimensional structures, it should be considered a heterostructure type $n-\mathrm{PbTe} / p-\mathrm{SnTe} / n-\mathrm{PbTe}$ [9]. There is a good correspondence between theoretical calculation of the oscillation period $\left(\Delta d_{\text {theor }}=3.0 \pm 0.4 \mathrm{~nm}\right)$ and that experimentally obtained $\left(\Delta d_{\exp }=2.9 \pm 0.3 \mathrm{~nm}\right)$, using the justified Fermi energy $0.5 \mathrm{eV}$ (Table) [9].

Note that analysing $d$-dependences for thermoelectric parameters of nanostructures, one should take into consideration the self-organization processes that determine its maxima $[11,12,16]$. These approaches to explanation of experimental data are vindicated by coincidence of the point extrema in thickness dependences of independently measured Hall $R_{\mathrm{H}}$ and Seebeck coefficients $S$ (Figs 2 and 3).

\section{Conclusions}

1. It has been shown that for PbTe nanostructures on polyamide dependences of conductivity, Hall factor, mobility, concentration, Seebeck and thermoelectric power coefficients on thickness demonstrate nonmonotonic, oscillatory behavior associated with dimensional quantization of the hole energy spectrum in quantum wells.

2. It has been established that with the concentration increase of the main type carrier (holes), there is a shift in extrema for thickness dependences of thermoelectric parameters to less values of the nanostructure thickness.

3. The model of a quantum well that implies the existence of two barriers - on the surface of $\mathrm{PM}-1 / p$ $\mathrm{PbTe}$ structure under influence of oxygen, which forms the acceptor centers enriched layer, and polyamide substrate, - is proposed.

4. The difference between experimental and theoretical (parabolicity of energy bands and infinite depth of the well) oscillation amplitude in thickness dependences of thermoelectric parameters explained by assumption of the infinite barrier height.

5. It has been revealed that the oscillation period of thermoelectric parameters coincides with the size of crystallites in $\mathrm{PM}-1 / p$-PbTe nanostructures. Energy parameters were determined for nanostructures based on compounds IV - VI .

\section{References}

1. M.S. Dresselhaus, G. Chen, M.I. Tang, R. Yang, H. Lee, New directions for low-dimensional thermoelectric materials // Adv. Mater. 19(1), p. 1043-1053 (2007).

2. L.D. Hicks, T.C. Harman, X. Sun, M.S. Dresselhaus, Experimental study of the effect of quantum-well structures on the thermoelectric figure of merit // Phys. Rev. B, 53, R10493 (1996).

3. M.S. Dreselhaus, Lin Yu-Ming, T. Koga, S.B. Cronin, O. Rabin, M.R. Black, G. Dresselhaus, Low dimensional thermoelectricity, in: Semiconductors and Semimetals: Recent Trends in Thermoelectric Materials Research III, ed T.M. Tritt, p. 1-121. Academic, San Diego, CA, 2001.

4. D.M. Freik, I.K. Yurchyshyn, V.V. Bachuk, L.T. Harun, Yu.V. Lysiuk, Thermoelectric properties of PbTe thin films during exposure to air // Physics and chemistry of solid state 11(3), p. 598-603 (2010).

5. E.I. Rogacheva, S.G. Lyubchenko, M.S. Dressel haus, Effect of oxidation on thickness dependences of thermoelectric properties in $\mathrm{PbTe} / \mathrm{mica}$ thin films // Thin Solid Films, 476(2), p. 391-395 (2005).

6. E.I. Rogacheva, I.M. Krivulkin, O.N. Nashchekina, A.Yu. Sipatov, V.V. Volobuev and M.S. Dressel haus, Effect of oxidation on the thermoelectric properties of $\mathrm{PbTe}$ and $\mathrm{PbS}$ epitaxial films // Appl. Phys. Lett. 78(12), p. 1661-1663 (2001).

7. E.I. Rogacheva, T.V. Tavrina, O.N. Nashchekina, S.N. Grigorov, K.A. Nasedkin, M.S. Dresselhaus, S.B. Cronin, Quantum size effects in $\mathrm{PbSe}$ quantum wells // Appl. Phys. Lett. 80(15), p. 26902692 (2002).

8. E.I. Rogacheva, O.N. Nashchekina, Y.O. Vekhov, M.S. Dresselhaus, S.B. Cronin, Effect of thickness on the thermoelectric properties of PbS thin films // Thin Solid Films, 423(1), p. 115-118 (2003).

9. E.I. Rogacheva, O.N. Nashchekina, A.V. Meriuts, S.G. Lyubchenko, M.S. Dresselhaus, G. Dresselhaus, Quantum size effects in n-PbTe/p-SnTe/nPbTe heterostructures // Appl. Phys. Lett. 86(6), p. 063103 (2005).

10. E.I. Rogacheva, O.N. Nashchekina, S.N. Grigorov, M.A. Us, M.S. Dresselhaus, S.B. Cronin, Oscillatory behaviour of the transport properties in PbTe quantum wells // Nanotechnology, 14(1), p. 53-59 (2003).

11. S.A. Chambers, Epitaxial film crystallography by high-energy Auger and X-ray photoelectron diffraction // Adv. Phys. 40(4), p. 357-415 (1991).

12. I. Markov, S. Stoyanov, Mechanisms of epitaxial growth // Contemp. Phys. 28(3), p. 267-320 (1987). 
13. M.P. Singh, C.M. Bhandari, Non-monotonic thermoelectric behavior of lead telluride in quantum-well-like structures // Solid State Communs. 133, p. 29-34 (2005).

14. A. Casian, I. Sur, H. Scherrer, Z. Dashevsky, Thermoelectric properties of n-type $\mathrm{PbTe} / \mathrm{Pb}_{1-\mathrm{x}} \mathrm{Eu}_{\mathrm{x}} \mathrm{Te}$ quantum wells // Phys. Rev. B, 61(23), p. 15965-15974 (2000).
15. Yu.N. Ravich, B.A. Efimova, N.A. Smirnova, Methods of Investigations of Semiconductors in Application to Lead Chalcogenides PbTe, PbSe, $\mathrm{PbS}$, ed. L.S. Stilbans. Nauka, Moscow, 1968 (in Russian), p. 384.

16. E.I. Rogacheva, I.M. Krivulkin, O.N. Nashchekina, A.Yu. Sipatov, V.A. Volobuev, M.S. Dresselhaus, Percolation transition of thermoelectric properties in PbTe thin films // Appl. Phys. Lett. 78(21), p. $3238-3240$ (2001). 